\title{
Age-Dependent Production and Replacement Strategies in Failure-Prone Manufacturing Systems
}

\author{
Samir Ouaret ${ }^{1}$, Jean-Pierre Kenné ${ }^{1}$, Ali Gharbi ${ }^{2}$ and Vladimir Polotski ${ }^{1}$
}

\begin{abstract}
A failure-prone manufacturing system that consists of one machine producing one type of product is studied. The random phenomena examined are machine breakdowns and repairs. We assume that the machine undergoes a progressive deterioration while in operation and that the machine failure rate is a function of its age. The aging of the machine (the dynamics of the machine age) is assumed to be an increasing function of its production rate. Corrective maintenance activities are imperfect and restore the age of the machine to as-bad-as-old conditions (ABAO). When a failure occurs, the machine can be repaired, and during production, the machine can be replaced, depending on its age. When the replacement action is selected, the machine is replaced by a new and identical one. The decision variables are the production rate and the replacement policy. The objective of this paper is to address the simultaneous production and replacement policy optimization problem in the context of manufacturing with deterioration and imperfect repairs satisfying the customer demand and minimizing the total cost, which includes costs associated with inventory, backlog, production, repair and replacement, over an infinite planning horizon. We thoroughly explore the impact of the machine aging on the production and replacement policies. Particular attention is paid to the verification of underlying mathematical results that guarantee the existence of optimal solutions and the convergence of numerical methods. Due to imperfect repairs, the dynamics of the system is affected by the system history and semi-Markov processes have to be used for modeling. Optimality conditions in the form of the Hamilton-Jacobi-Bellman (HJB) equations are developed, and numerical methods are used to obtain the optimal control policies (production (rate) and replacement policies). A numerical example is given to illustrate the proposed approach, and an extensive sensitivity analysis is presented to confirm the structure of the obtained control policies.
\end{abstract}

Manufacturing systems, optimal control, replacement policy, corrective maintenance, production planning, numerical methods

\section{Introduction}

In the manufacturing environment, the availability of the machine often decreases due to its age and also to imperfect maintenance activities. In general, corrective or preventive maintenance brings the state of the machine to a level which is not new, and it may not be able to meet the demand rate for the commodity produced. For this reason, the machine has to be replaced. We consider a machine that is subject to random breakdowns and repairs. It undergoes deterioration while in operation, and the failure rate increases with its age. The aging of the machine is an increasing function of its production rate. The corrective maintenance activities performed are imperfect and restore the machine to as-bad-as-old conditions. Replacement activities for their part renew the machine, which is similar to restoring the machine to as-good-as-new conditions (resetting its age to zero).

The first objective of this paper is to simultaneously determine production and replacement policies in a manufacturing environment under deterioration and imperfect repairs. We enhance existing mathematical models by including the production cost in the objective function. Given that the dynamics of the machine aging process depends on the production rate, penalizing the latter helps to amplify the impact of aging and push the system (optimal control policies) towards an appropriate solution. The proposed model appears to be better at addressing industrial reality, and is yet to be used in the literature in analyzing age-dependent production and replacement strategies. The obtained solution provides the simultaneous optimal control of production and replacement of the machine (assuming that one replacement is performed). The decision variables are the production rate and the replacement policy. The dynamics of the machine is described by a semi-Markov decision model due to the machine's deterioration and imperfect repairs (as-bad-as-old). The optimal control policies are determined in order to satisfy a deterministic customer demand and minimize inventory, backlog, production, repair and replacement costs over an infinite planning horizon.

\footnotetext{
${ }^{1}$ Mechanical Engineering Department, École de technologie supérieure, University of Quebec, Montreal, Quebec, Canada

${ }^{2}$ Automated Production Engineering Department, École de technologie supérieure, University of Quebec, Montreal, Quebec, Canada

Corresponding author:

Vladimir Polotski, Mechanical Engineering Department, École de technologie supérieure, University of Quebec, 1100 Notre-Dame Street West, Montreal, Quebec, Canada, H3C 1K3.

Email: vladimir.polotski@etsmtl.ca
} 
The second objective is to consider machine aging as a unique factor affecting the deterioration of the machine in order to get a better understanding of the phenomena specifically relating to machine aging without interference from other deterioration levels. Finally, the third objective is to provide a rigorous formulation of the underlying mathematical results and verification of all conditions in order to guarantee the existence of optimal solutions and convergence of numerical methods used to find these solutions.

The rest of the paper is organized as follows. We provide the overview of the existing body of works in the "literature review" section. In the "Assumptions and problem statement" section, we describe the assumptions regarding the model and formulate the optimal control problem. The "Properties of the value function and optimality conditions" section addresses optimality conditions in the form of HJB equations. A numerical example is presented in the "Numerical example" section, followed in the "Sensitivity analysis" section by the sensitivity analysis illustrating the robustness and effectiveness of the obtained control policies. Discussions of the results are presented in "Discussions" section and finally, the paper is summarized in the "Conclusion" section.

\section{Literature review}

To describe the behaviour of a repairable system subject to failures, various types of repairs are used in conventional models: the minimal repair, the perfect repair and the imperfect repair. The first one brings the system to its functioning condition just prior to a failure (e.g. as-bad-asold); the second one is as-good-as-new, while the third one can be represented by its so-called virtual age, which is smaller than the real age. Pham and Wang ${ }^{1}$ suggest the faulty part being only partially repaired, human errors such as further damage during maintenance, replacement with faulty parts, etc., as reasons behind an imperfect repair. Chiu et al. ${ }^{2}$ For their part address system failures followed by imperfect rework using economic manufacturing quantity model with Poisson failure and abort/resume control policies. Many authors have studied the optimization problems of maintenance, repair and replacement in the context of a progressive deterioration, but without addressing production planning. Progressive deterioration is usually characterized either by the repair time increasing with the number of failures or by age accumulation when the machine is in operational mode. Phelps ${ }^{3}$ developed a semi-Markov decision model in which a minimal repair is performed on a system subject to random failures. He suggested three optimal replacement policies: in the first policy, the system is replaced at a fixed time $\mathrm{T}$; in the second, the system is replaced after a fixed number of failures $\mathrm{N}$; and in the third, the replacement takes place at the first failure after a fixed time T. Kijima et al. ${ }^{4}$ studied a problem of periodic replacement with a general repair: the system is replaced only at a regular time interval $\mathrm{kT}$ $(\mathrm{k}=0,1, \ldots)$, and is repaired following a failure. They assumed that the costs of repair and replacement are constant. This general repair transfers the system state to a certain "better" state. A stochastic model describing this situation was proposed to find the optimal replacement period. Makis and Jardine $^{5}$ described a system with deterioration subject to random failures modeled as a semi-Markov process, and demonstrated under appropriate conditions that a stationary optimal replacement policy exists. They assumed that the replacement cost is constant and that the repair cost depends on the number of failures and the real machine age. Love et al. ${ }^{6,7}$ proposed a discrete semi-Markov model: they determined the optimal policies of a machine subject to breakdowns, and which can be replaced or can undergo an imperfect repair. The virtual machine age has been introduced in the decision process, and it has been shown that the optimal repair/replacement policy is of threshold type. Zhang ${ }^{8}$ developed a bivariate replacement policy $(\mathrm{T}, \mathrm{N})$ for a repairable system. The replacement is performed at the first failure either after the cumulative age reaches $\mathrm{T}$ or the number of failures reaches $\mathrm{N}$. It was assumed that the system after repair is not refurbished, and that the optimal solution can be obtained analytically or numerically under certain conditions.

Many researchers have studied the production planning problem for unreliable manufacturing systems. Based on the formalism of Rishel, ${ }^{9}$ Olsder and Suri $^{10}$ determined the optimum conditions of a planning problem in the production of a manufacturing system subject to random breakdowns and repairs, with its dynamics described by homogeneous Markov processes. The optimum conditions are described by the HJB equations. The pioneering work of Kimemia and Gershwin ${ }^{11}$ similarly showed that the obtained control policy for this problem is of threshold type (Hedging point policy). This allows the optimal production rate to be found in order to meet a constant demand rate and reduce the total cost, which is the sum of inventory and shortage costs. Boukas and Haurie ${ }^{12}$ determined a policy that combines the production and preventive maintenance for a manufacturing system consisting of two machines. They consider that the probability of failures of the machines increases with the age. Since the repair or preventive maintenance activities restore the cumulative age to zero, the dynamics of the system (machines) is modeled in Boukas and Haurie ${ }^{12}$ by a non-homogenous Markov process. The set of dynamic programming equations were solved numerically based on Kushner's approach, ${ }^{13}$ and the obtained control policy is of modified threshold type. Yan ${ }^{14,15}$ discussed a hierarchical stochastic production planning problem of flexible manufacturing systems (FMSs). Imposing some conditions on uncertain demand, the author formulated a stochastic non-linear programming problem and proposed the algorithms for its solution. By applying some approximations, a production plan with the lowest possible cost is obtained.

In the context of imperfect repairs, the optimal production and repair/replacement joint policy is of great importance for practitioners. It helps to better manage the manufacturing system performance, and to intervene with the acquisition of a new machine in a timely fashion. This situation was investigated in Nodem et al. ${ }^{16}$ where the authors proposed a 
hierarchical decision making approach to first determine the hybrid repair and replacement policy $(T, N)$. Secondly, the optimal production rate is determined. The age and the number of failures are combined to make the decision about the repair/replacement of the machine. The repair activities are imperfect due to a number of failures affecting the system behaviour, and the replacement activities restore the age of the machine to initial conditions (AGAN). With respect to the modeling, the decision to repair or replace the machine is made when the failure occurs. In Gomez et al., ${ }^{17}$ the simultaneous production, repair/overhaul (equivalent to replacement) and preventive maintenance control policy is obtained under the effect of deteriorations resulting in product quality degradation: the authors combined the impact of two factors, namely, the wear of the machine and human interventions. When the machine is in operational mode, three types of actions can be taken: the machine produces, is sent to overhaul, or is sent for preventive maintenance. Upon failure, the machine has to be repaired. A semi-Markov process is used in both, ${ }^{16,17}$ due to the imperfect maintenance activities of the machine that depend on the history of breakdowns and repairs. The authors ${ }^{16,17}$ developed the optimality conditions in the form of HJB equations, that allow the resolution of the problem of stochastic optimal control based on stochastic dynamic programming and numerical methods, in order to optimize the total cost (e.g. including the inventory, backlog, repair/replacement, preventive maintenance and defectives costs) and satisfy a demand assumed to be deterministic and known. However, due to the mixture of various deterioration mechanisms involved in the proposed models, it remained unclear which mechanism was the most responsible for the phenomena observed.

As mentioned in the introduction, a model that differs from the existing ones is developed in this paper. We explore the impact of deterioration mechanism related to the machine aging only, in order to eliminate interference from other sources of deterioration, as in Nodem et al. ${ }^{16}$ and Gomez et al. ${ }^{17}$ and get the insight to age-induced deterioration phenomena. We also incorporate the production cost in the objective function. The rationale behind changing the cost function in such a way, given that the dynamics of the machine aging depends on the parts produced, penalizing the production rate with production cost will amplify the phenomenon of aging and push the system (optimal control) to enhance the obtained solution in manufacturing environment. The optimality conditions are developed in the form of $\mathrm{HJB}$ equations using the optimal control theory based on stochastic dynamic programming. The practical implication of the proposed model is verified through numerical simulations.

\section{Assumptions and problem statement}

This section defines the assumptions used throughout this article, as well as the optimal control problem statement.

\section{Assumptions}

The following assumptions are considered in this paper:

1. the raw materials are always available and unlimited;

2. the customer demand is known and described by a constant rate over time;

3. the maximum production rate of each machine is known;

4. the repair or replacement costs of the manufacturing machine are assumed constant ((\$/repair); (\$/replacement));

5. the machine deteriorates in operational mode, in which the machine age is continuously growing;

6. the failure rate of the machine increases with its age;

7. the corrective maintenance activities are imperfect and associated with a minimal repair that restores the machine to as-bad-as-old conditions (ABAO);

8. the machine is replaced by a new identical one;

9. the production is more penalized when the machine increases its production rate.

\section{Problem formulation}

The manufacturing system under consideration consists of one machine producing one type of product. The machine is unreliable, and is subject to random phenomena, such as breakdowns and repairs. The machine deteriorates while in operation, and its failure rate increases with its age. In failure, the machine undergoes a minimal repair, and while it is in operation, a decision can be made to replace the machine with a new and identical one due to its deterioration. A service inventory is built at the end of the production process, which is used to satisfy a constant demand rate. This situation is illustrated in Figure 1.

The system behaviour can be mathematically modeled by a stochastic control system in continuous time (not in multiperiod time, as is normally used in the literature) characterized by a hybrid state, which is comprised of two continuous state variables (inventory level $x(t)$ and machine age $a(t))$ and one discrete state variable (mode of the machine $\xi(t)$ ) at time $t$.

The dynamics of the stock level is described by a onedimensional ordinary differential equation:

$$
\begin{aligned}
& \dot{x}(t)=u(t)-d \\
& \text { with } t \in \mathbb{R}_{+} \text {and } x(0)=x^{0}
\end{aligned}
$$

where $x^{0}$ is the given initial inventory/backlog level. When $x(t) \geq 0$, the system has an inventory and a backlog, otherwise.

The cumulative age of the machine at time $t$, is the solution of the following differential equation:

$$
\dot{a}(t)=f(u(t))
$$

where $f($.$) is a positive real-valued function.$

In the case considered herein, the machine age $a(t)$ is the number of produced parts, and the aging of the machine $\dot{a}(t)$ will always be an increasing function of the machine production rate. By referring to the literature, e.g. (see Boukas 
and Haurie and Kenné and Boukas), ${ }^{12,18}$, we can use the linear model that describes the relationship between the aging of the machine $\dot{a}(t)$ and its production rate $u(t)$. That is:

$$
\dot{a}(t)=k u(t)
$$

with $t \in \mathbb{R}_{+}$and

$$
a(0)=a^{0}, a\left(T^{+}\right)=a\left(T^{-}\right) \text {and } a(T)=0
$$

where $k$ is a given positive constant and $a^{0}, T^{+}, T^{-}$and $T$ stand for the initial age, the last times of repair, operation and replacement of the machine, respectively. These values $\left(T^{+}\right.$, $T^{-}, T$ ) imply that the repair is as-bad-as-old and the replacement is as-good-as-new.

The production rate $u(t)$ must satisfy the capacity constraint:

$$
0 \leq u(\cdot) \leq u_{\max }
$$

where $u_{\max }$ is the maximum production rate of the manufacturing machine.

The machine's mode can be classified as operational, denoted by $\xi(t)=1$, under repair, denoted by $\xi(t)=2$ and under replacement, denoted by $\xi(t)=3$. The mode of the machine at time $t$ is given by the random process $\{\xi(t) \geq 0\}$ with $\xi(t) \in B=\{1,2,3\}$ such that:

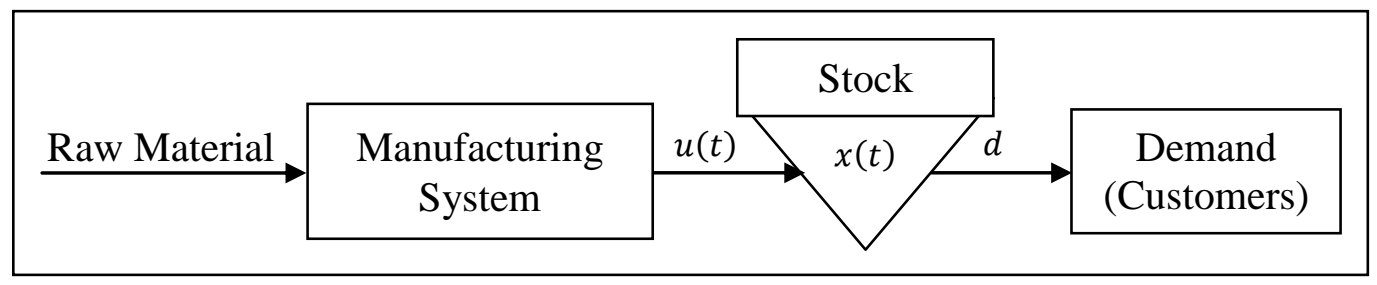

Figure 1. Structure of the production system.

$$
\xi(t)= \begin{cases}1 & \text { operational } \\ 2 & \text { under failure } \\ 3 & \text { under replacement }\end{cases}
$$

Given that the machine deteriorates with age and the fact that it is not new after repair activities (ABAO), its dynamics is modeled as a semi-Markov process in continuous time discrete state over an infinite horizon $\{\xi(t) \geq 0\}$. The transition diagram of such a process is illustrated in Figure 2. We introduce a control variable $w(t) \in\{0,1\}$ initially as a binary variable; it is set to a value 1 if the replacement of the machine is performed and to 0 otherwise. The transition rate $q_{13}($.$) is defined as a linear function of w(t): q_{13}()=.q \cdot w(t)$ where $q$ is a given constant.

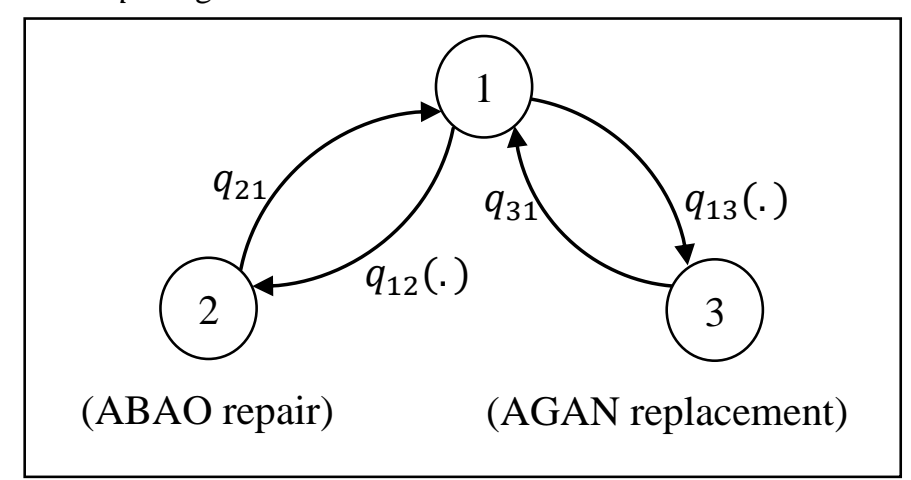

Figure 2. States transition diagram of the considered stochastic process.

The failure rate $q_{12}($.$) is an increasing function of a$ machine's age $a(t)$, and is given by:

$$
q_{12}(a(t))=A_{0}+A_{1}^{\infty}\left(1-e^{-\left(A_{2} a(t)^{3}\right)}\right)
$$

where the parameters $A_{0}, A_{1}^{\infty}$ and $A_{2}$ are given constants. The expression given by equation (5) describes the impact of a machine age on its dynamics, as in Kenné and Nkeungoue. ${ }^{19}$ The inverse of the transition rate $q_{12}(a)$ represents the mean time to failure as a function of the age denoted by $\operatorname{MTTF}(a)$. We use the notation $\operatorname{Ind}\{\Theta()$.$\} for the indicator function of a$ condition $\Theta($.$) defined as follows:$

$$
\operatorname{Ind}\{\Theta(.)\}= \begin{cases}1 & \text { if } \Theta(.) \text { is true } \\ 0 & \text { otherwise }\end{cases}
$$

The machine's mode switches from operational to replacement with a transition rate denoted by $q_{13}($.$) , the$ inverse of $q_{13}($.$) represents the expected delay between the$ preparation of the order of the new machine, its installation kit and its arrival on the site. In order to allow this delay to vary continuously, we reintroduce a control variable $w(t)$ allowing it to take its values within the domain ]0,1]. We also assume that the decision regarding the machine replacement can be taken only in operational mode. Thus, the delay corresponding to the inverse of $q_{13}()=.q . w(t)$ is the transition time between the decision to perform the replacement and the effective switch from the operational mode to the replacement mode. The replacement is now allowed for all possible values of $w(t)$ within $] 0,1]$. When the value of $w(t)$ is close to zero (e. g. $10^{-5}$ ), the delay before replacement is very large (but finite), thus describing the situation when replacements do not actually take place; as for machine repairs, they can occur at any failure instant. The transition rates $q_{21}$ to repair the machine in mode 2 and $q_{31}$ to replace the machine in mode 3 are assumed to be known constants (their inverses represent the mean time to repair and the mean time to replace, respectively). Other transition rates of the manufacturing system are equal to zero. 
In the case of minimal repair, the repair rate is usually considered to be constant. In manufacturing systems, many repairs such as (as-bad-as-old) can be considered as minimal repairs. Such a repair brings the age of the machine in the state which is basically the same as it was just before the failure occurred. In other words, a minimal repair means that the age of the machine is not affected by failures: the machine age after each minimal repair is restored, and the failure rate gets the same value as it was before the failure. Thus, after minimal repair, both failure rate and repair rate are restored to their values before the last failure; $q_{12}^{\text {new }}=q_{12}^{\text {old }}$, and $q_{21}^{\text {new }}=q_{21}^{\text {old }}$. The ratio of repair duration $\tau_{r}$ and the mean time to failure $1 / q_{12}$ are then kept constant in the case of minimal repair and then:

$\tau_{r}^{\text {new }}=\tau_{r}^{\text {old }} \cdot \frac{q_{12}^{\text {old }}}{q_{12}^{\text {new }}}=\tau_{r}^{\text {old }} \cdot \frac{q_{12}^{\text {old }}}{q_{12}^{\text {old }}} \Rightarrow \tau_{r}^{\text {new }}=\tau_{r}^{\text {old }}$

As a result, the repair duration $\tau_{r}$ is represented by the constant repair rate $q_{21}$. More detailed description of the minimal repair models can be found in Zio. ${ }^{20}$ For example: once the machine is repaired after the first failure, the age continues to accumulate when the machine begins to operate again and the failure rate continues also to increase with the age. When the next failure occurs, and a minimal repair is executed, the failure rate gets the same value as before the last failure and according to the equality above $\left(\tau_{r}^{\text {new }}=\tau_{r}^{\text {old }}\right)$, the repair duration does not change; it is the same as before the failure.

Figure 3 shows the jump times occurring within our model in the different modes $e_{i}$. It should be recalled that the mode of the manufacturing system at time $t$ is given by the finite-state semi-Markov process $\xi(t) \in B=\{1,2,3\}$, and can be characterized by the matrix $Q(w)=\left[q_{\alpha \beta}().\right]$. Its entries $q_{\alpha \beta}$ are real numbers, and depend on the decision variable $w($.$) ,$ with $\alpha, \beta \in B$. If $\alpha \neq \beta$ we have

$$
\begin{aligned}
& q_{\alpha \beta}(x, a, u, w) \geq 0 \\
& \sum_{\beta} q_{\alpha \beta}(x, a, u, w)=0
\end{aligned}
$$

and if $\alpha=\beta$, we have

$$
q_{\alpha \alpha}(x, a, u, w)=-\sum_{\alpha \neq \beta} q_{\alpha \beta}(x, a, u, w)
$$

The transition probabilities of the manufacturing system from mode $\alpha$ to mode $\beta$ after time $t$ are given by:

$$
\begin{aligned}
P[\xi(t+\delta t) & =\beta \mid \xi(t)=\alpha, a(t)=a, u(t)=u, w(t)=w] \\
& =q_{\alpha \beta}(x, a, u, w) \delta t+o(x, a, \delta t) \\
P[\xi(t+\delta t) & =\alpha \mid \xi(t)=\alpha, a(t)=a, u(t)=u, w(t)=w] \\
& =1+q_{\alpha \alpha}(x, a, u, w) \delta t+o(x, a, \delta t)
\end{aligned}
$$

where $o(x, a, \delta t)$ is a quantity such that:

$$
\lim _{\delta t \rightarrow 0} \frac{o(x, a, \delta t)}{\delta t}=0
$$

for all $\alpha, \beta \in B$

The corresponding $(3 \times 3)$ transition matrix of the semiMarkov chain $\xi(t)$ for the considered system is given by:

$$
Q(w)=\left[\begin{array}{ccc}
q_{11} & q_{12}(a(t)) & q_{13}(.) \\
q_{21} & q_{22} & 0 \\
q_{31} & 0 & q_{33}
\end{array}\right]
$$

To address the feasibility of the manufacturing system, we introduce as usual, the limiting probabilities $\pi_{i}$ of mode $i$, $i \in\{1,2,3\}$ that are known to be steady state solutions of the forward Kolmogorov equations $\dot{\pi}(t)=\pi(t) Q(t)$. When $t \rightarrow \infty, \dot{\pi}(t)=0$, as in Ross. ${ }^{21}$ Therefore, we have:

$$
\pi(.) Q(.)=0
$$

with the normalizing condition:

$$
\sum_{i=1}^{3} \pi_{i}=1
$$

where $\pi()=.\left(\pi_{1}, \pi_{2}, \pi_{3}\right)$ and $Q($.$) is a (3 \times 3)$ transition rates matrix given by equation (11). The manufacturing system is considered feasible if:

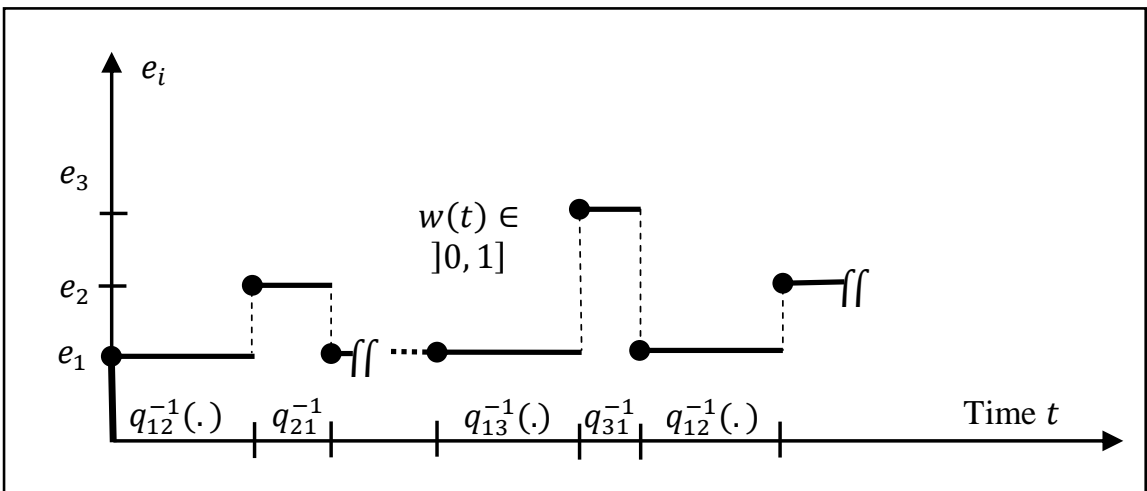

Figure 3. Jump times of different modes $e_{i}(i=1,2$ and 3$)$. 


$$
\pi_{1} u_{\max } \geq d
$$

Let $G($.$) be the cost rate defined as follows:$

$$
G(\alpha, x, a, u, w)=c^{+} x^{+}+c^{-} x^{-}+c^{\alpha}+c(u)
$$

The constants $c^{+}$and $c^{-}$are costs incurred per unit of produced parts and per unit time, and are used to penalize parts inventory and backlog, respectively, with $x^{+}=\max (0, x)$, $x^{-}=\max (0,-x)$ and $c^{\alpha}$ being a constant defined as follows:

$$
c^{\alpha}=c_{r} \cdot \operatorname{Ind}\{\xi(t)=2\}+c_{0} \cdot q_{31} \cdot \operatorname{Ind}\{\xi(t)=3\}
$$

where $c_{r}$ is the cost rate (positive constant) for repair activity on the machine and $c_{0} . q_{31}$ is the replacement cost (positive constant) depends on the duration of the replacement activity of the machine, respectively. Let $c(u)$ be a linear production cost function given by $c(u)=c_{u} u$. The constant $c_{u}$ is the cost incurred per unit of produced parts, and used here to penalize the production rate of the machine.

The objective of this research is to find the two decision variables, namely, the production rate $u($.$) and the$ replacement variable $w($.$) , that would minimize the expected$ discounted cost $J($.$) given by:$

$$
\begin{aligned}
& J(\alpha, x, a, u, w)=E\left\{\int_{0}^{\infty} e^{-\rho t} G(.) d t \mid \xi(0)=\right. \\
& \alpha, x(0)=x, a(0)=a\} \forall(u(.), w(.)) \in \Gamma(\alpha)
\end{aligned}
$$

where $\rho$ is the discount rate, and $(\alpha, x, a)$ are the initial values of the state variables. The set of admissible decisions $\Gamma(\alpha)$ that define the feasible plan $(u(a,),. w(a,)$.$) depends on the$ stochastic process $\xi(t)$, and is given by:

$$
\Gamma(\alpha)=\left\{\begin{array}{c}
(u(a, .), w(a, .)) \in \mathbb{R}^{2}, 0 \leq u(a, .) \leq \\
\left.u_{\max } \operatorname{Ind}\{\xi(t)=1)\right\}, 0<w(a, .) \leq 1
\end{array}\right\}
$$

The value function of such a problem is given by:

$$
v(\alpha, x, a)=\inf _{(u(a,), w(a,)) \in \Gamma(\alpha)} J(\alpha, x, a, u, w)
$$

$\forall \alpha \in B, x \in \mathbb{R}, a \in \mathbb{R}_{+}$

The properties of the value function leading to HJB equations of the stochastic optimal control problem are presented in the next section.

\section{Properties of the value function and optimality conditions}

In this section, we develop the optimality conditions using the optimal control theory based on stochastic dynamic programming. The optimal control policy $\left(u^{*}(),. w^{*}().\right)$ denotes a minimizer over $\Gamma(\alpha)$ on the right hand side of equation (16). This policy shows that the value function $v($. given by equation (18) satisfies the set of partial differential equations known as the Hamilton-Jacobi-Bellman equations (HJB). In Appendix 2, we develop the derivation of the HJB equations. However, the differentiability and viscosity properties of the value function can be found in Kenné et al. ${ }^{22}$ From equation (15), $G(\alpha, a, x, u, w)$ can be written as follows: $G(\alpha, z, U)=h(x)+c^{\alpha}+c(u)$, where $z=(x, a)$ and $U=(u, w)$. We extend the domain of the decision variable $w$ serving to describe the controlled replacement to a continuous domain ]0,1], in order to use the notions of continuity and convexity. This however will not affect the optimization result since for the type of problem we are solving, the optimum (over $w$ ) always occurs on the boundary of ]0,1]. We should recall that in our case, the replacement is allowed for all possible values of the variable $w$. Below, we will use the following assumption, definition and Lemmas.

Assumption A1. $Q(w)$ is a continuous function in $w$.

Lemma 1.

1. $G(\alpha, z, U)$ is jointly convex if, for each $\alpha \in B$ and for every $z_{1}, z_{2}, U_{1}$ and $U_{2}$ and $\delta \in[0,1]$, e.g.

$$
\begin{aligned}
G\left(\alpha, \delta z_{1}\right. & \left.+(1-\delta) z_{2}, \delta U_{1}+(1-\delta) U_{2}\right) \\
\leq & \delta G\left(\alpha, z_{1}, U_{1}\right)+(1-\delta) G\left(\alpha, z_{2}, U_{2}\right)
\end{aligned}
$$

Then $v(\alpha, z)$ is also convex if, for each $\alpha \in B$ and for every $z_{1}$ and $z_{2}$ and $\delta \in[0,1]$, e.g.

$$
v\left(\alpha, \delta z_{1}+(1-\delta) z_{2}\right) \leq \delta v\left(\alpha, z_{1}\right)+(1-\delta) v\left(\alpha, z_{2}\right)
$$

Moreover, $G(\alpha, z, U)$ is strictly jointly convex in $z$ if the inequality above of $G$ holding as an equality for some $0<\delta<$ 1 implies $z_{1}=z_{2}$ and $U_{1} \neq U_{2}$. Then $v(\alpha, z)$ is also strictly convex in $z$ if the inequality above of $v$ is strict whenever $z_{1} \neq z_{2}$ and $0<\delta<1$.

2. For some constants $C_{g}$ and $K_{g}>0$, if $G(\alpha, z, U)$ is locally Lipschitz in $z$, e.g.

$$
\begin{aligned}
\left|G\left(\alpha, z_{1}, U\right)-G\left(\alpha, z_{2}, U\right)\right| & \\
\leq & C_{g}\left(1+\left|z_{1}\right|^{K_{g}}+\left|z_{2}\right|^{K_{g}}\right)\left|z_{1}-z_{2}\right|
\end{aligned}
$$

Then $v(\alpha, z)$ is also locally Lipschitz in $z$, e.g.

$$
\left|v\left(\alpha, z_{1}\right)-v\left(\alpha, z_{2}\right)\right| \leq C_{g}\left(1+\left|z_{1}\right|^{K_{g}}+\left|z_{2}\right|^{K_{g}}\right)\left|z_{1}-z_{2}\right|
$$

Proof. Any function which is convex and locally Lipschitz is continuously differentiable. To prove this property for the value function $v(\alpha, z)$ with respect to $z$ and for each $\alpha \in B$, we can refer the reader to the book by Sethi et al., ${ }^{23}$ and the proof is similar to their proof of Lemma E.1.

The value function $v(\alpha, \mathrm{z})$ is continuously differentiable in $z$ if and only if $D^{+} v(\alpha, z)$ and $D^{-} v(\alpha, z)$ are both singletons. In this case:

$$
D^{+} v(\alpha, z)=D^{-} v(\alpha, z)=\left\{v_{z}(\alpha, z)\right\}
$$

where $z=(x, a)$. The HJB equations corresponding to the optimal control problem are written as follows: 


$$
\begin{aligned}
& \rho v(\alpha, x, a) \\
& =\min _{(u, w) \in \Gamma(\alpha)}\left[\begin{array}{c}
G(\alpha, x, a, u, w)+(u-d) \frac{\partial}{\partial x} v(\alpha, x, a) \\
+k u \frac{\partial}{\partial a} v(\alpha, x, a) \\
+\sum_{\beta} q_{\alpha \beta}(.) v\left(\beta, x, \varphi_{a}(\xi, a)\right)
\end{array}\right]
\end{aligned}
$$

where $\varphi_{a}(\xi, a)$ is the function that restores the age of the machine to as-good-as-new after the machine is replaced, and to as-bad-as-old after imperfect repair activities. Therefore, at a jump time $\tau$ for the process $\xi$ one can write this function as follows:

$$
\varphi_{a}(\xi, a)=\left\{\begin{array}{cc}
a\left(\tau^{-}\right) & \text {if } \xi\left(\tau^{+}\right)=1 \text { and } \xi\left(\tau^{-}\right)=2 \\
0 & \text { if } \xi\left(\tau^{+}\right)=1 \text { and } \xi\left(\tau^{-}\right)=3 \\
a\left(\tau^{-}\right) & \text {otherwise }
\end{array}\right.
$$

where $\xi(t)=\alpha \in B$, and $\partial v(\alpha, x, a) / \partial x$ and $\partial v(\alpha, x, a) / \partial a$ are the first-order partial derivatives of the value function $v(\alpha, x, a)$ with respect to $x$ and to $a$, in that order. The following definition will be used to prove the Lemma given below.

Definition 1. The superdifferentiability $D^{+} f(x)$ and subdifferentiability $D^{-} f(x)$ of any function $f(x)$ with respect to $x$ are defined as follows:

$$
\begin{aligned}
& D^{+} f(x)=\left\{s \in \mathbb{R}^{n}: \lim _{h \rightarrow 0} \sup \frac{f(x+h)-f(x)-h . s}{|h|} \leq 0\right\} \\
& D^{-} f(x)=\left\{s \in \mathbb{R}^{n}: \lim _{h \rightarrow 0} \inf \frac{f(x+h)-f(x)-h \cdot s}{|h|} \geq 0\right\}
\end{aligned}
$$

Lemma 2. The value function $v($.$) defined in equation (18) is$ the unique viscosity solution to the HJB equations (19).

Proof. To prove the Lemma 2, we use the concept given by Definition 1 to extend Theorem 2 presented by Yan and Zhang. ${ }^{24}$

When the value function is available, an optimal control policy can be obtained as a solution of the HJB equations (19). Since an analytical solution is impossible to obtain in general, it is a common practice to develop numerical methods for solving the HJB equations. Boukas and Haurie ${ }^{12}$ implemented a numerical method initially introduced by Kushner and Dupuis ${ }^{13}$ to solve such a problem in the context of production planning. Kushner's method used to solve the proposed optimality conditions is presented in Appendix 2. In the subsequent section, we provide a numerical example to illustrate the structure of the control policies.

\section{Numerical example}

The computational domain of the state variables is defined as $G_{x a}=G_{x}^{h} \times G_{a}^{h}$ such that:

$$
\begin{aligned}
& G_{x}^{h}=\{x:-10 \leq x \leq 30\} \\
& G_{a}^{h}=\{a: 0 \leq a \leq 100\}
\end{aligned}
$$

with $h_{x}=0.5$ and $h_{a}=2$. The imperfect repair is characterized by a minimal repair which brings the machine to as-bas-as-old conditions. The machine failure rate is assumed to be age-dependent. Using the model of the failure rate given by equation (5), we can obtain its trajectory according to the machine age, as illustrated in Figure 4 with values of $A_{0}=$ $10^{-4}, A_{1}^{\infty}=0.01$ and $A_{2}=5 \times 10^{-6}$.

Other parameters needed in the numerical example are presented in Table 1. These parameters have been chosen such as to ensure that the system is feasible. Using the formula of equation (14), the feasibility of the system was verified and respected on the all computational domains of the age, where $\left(q_{21}^{-1}, q_{31}^{-1}, q_{13}^{-1}\right)$ are the mean time to repair, the mean time to replace and the mean time between replace of the machine, respectively.

The production rate and replacement policy are presented in Figures 5 and 6, respectively. We can see that the obtained optimal production control policies can be viewed as an extension of the hedging point policy presented in Akella and Kumar. $^{25}$ In our case, however, we take into account the effect of the deterioration of the machine along the production phase, as well as the imperfect repairs. The optimal production control policy presented in Figure 5, and defined by $Z(a)$, which shows the numbers of parts to hold in inventory in order to hedge against failures of the machine with the age $a$. This control policy consists of the following rule:

- $\quad$ if the current stock level $x(t)$ is under the threshold level value $Z(a)$, the production rate is to be set to its maximum value;

- if it is exactly at the threshold level, the production rate is to be set to the demand rate, and

- if it is above the threshold level, the production rate is to be set to zero (produce nothing).

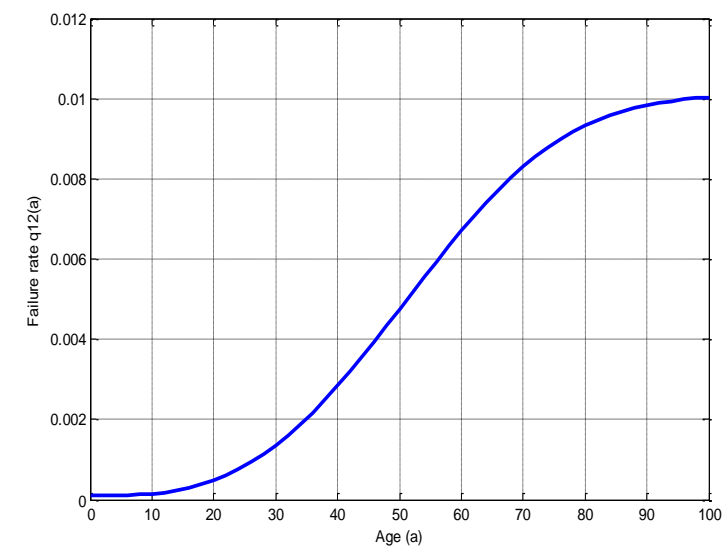

Figure 4. Age-dependent failure rate of the machine. 
Table 1. Parameters of the numerical example.

\begin{tabular}{llllll}
\hline Parameter & $c^{+}$ & $c^{-}$ & $c_{r}$ & $c_{0}$ & $c_{u}$ \\
Unit & $(\$ /$ product/time unit) & $(\$ /$ missing product/time unit) & $(\$ /$ time unit) & $(\$ /$ replacement $)$ & $(\$ /$ product $)$ \\
Value & 10 & 150 & 25 & 3000 & 100 \\
Parameter & $u_{\max }$ & $d$ & $\rho$ & $k$ & $w$ \\
Unit & (products/time unit) & (products/time unit) & & 0.8 & ] $0,1]$ \\
Value & 0.55 & 0.4 & 0.01 & & \\
Parameter & $q_{21}^{-1}$ & $q_{13}^{-1}$ & $q_{31}^{-1}$ & & \\
Value & 20 & 45 & 14 & & \\
\hline
\end{tabular}

Thus, the production control policy satisfies:

$$
u^{*}(1, x, a)= \begin{cases}u_{\max } & \text { if } x(t)<Z(a) \\ d & \text { if } x(t)=Z(a) \\ 0 & \text { if } x(t)>Z(a)\end{cases}
$$

where $Z(a)$ is the machine-age dependent function that gives the optimal production threshold in operational mode for each value of age of the machine, as illustrated in Figure 5.

We could not prevent the machine from getting older because only minimal repair (ABAO) is possible. Therefore, the machine deteriorated further, and after a certain age, could no longer satisfy the demand. Thus, a replacement policy defines when to replace the machine, taking into account the required stock level allowing the demand rate to be met when the machine is sent for replacement. In other words, the problem to be solved is determining the levels $\left(x^{*}, a^{*}\right)$ at which the machine should be replaced with a new identical one in order to optimize the manufacturing system.

Figure 6 shows that the optimal control for the replacement policy is a bang-bang solution. The reason for this is that the optimal control switches from the upper bound to the lower bound, and is restricted somewhere between the two. Let $w(1, x, a)$ denotes a switching function based on the stock level $x(t)$ and the age of the machine $a(t)$, with the maximum value $w_{\text {max }}=1$ if the machine must be sent for a replacement activity, and the minimum value (lower value) $w_{\min }=10^{-5}$ if the option to replace the machine is not recommended. We should recall that we only consider the decision process until the first replacement. Thus, the replacement policy can be written as follows:

$$
w^{*}(1, x, a)=\left\{\begin{array}{cc}
w_{\max } & \text { if }(x(t), a(t)) \in \text { zone } B \\
w_{\min } & \text { otherwise }
\end{array}\right.
$$

where states variables $(x(t), a(t))$ are the parameters of zone $B$. We can also see that Figure 6 shows the behaviour of the decision variable responsible for replacement, based on the machine age and inventory level. To better understand the obtained policy we divide the plane $(x, a)$ into two zones, A and $\mathrm{B}$.

Zone A: the replacement policy does not recommend sending the machine for replacement and the inventory level is low.
The machine is still new in this zone, and able to satisfy the demand with a rare fear of failure. Thus, it is not necessary to build a significant inventory level. This result is in a good agreement with the "zero inventory condition" of Bielecki and Kumar, ${ }^{26}$ which asserts that a zero-inventory policy can be exactly optimal even in the presence of uncertainty. Hence, the decision variable $w($.$) is set to its minimum value, (w()=$. $10^{-5} \approx 0$ ).

Zone B: the machine is aging and the failure rate increases. Here, the replacement cost is justified, and performing a replacement becomes necessary. However, before that is done, the manufacturing system must ensure a certain inventory level to satisfy the demand, and to hedge against possible failures and a non-productive replacement time. In this case, the decision variable $w($.$) is set to its maximum value, (w()=$. 1).

To better illustrate the optimal production and replacement policies, we can use their boundaries defined by $Z(a)$ in Figure 5 and $D(a)$ in Figure 6, respectively. The control policy defined by Figure 7 is known as the switching curve policy, and is characterize by a vector $z$ of two threshold parameters $z=(Z(a), D(a))$, the optimal production and replacement

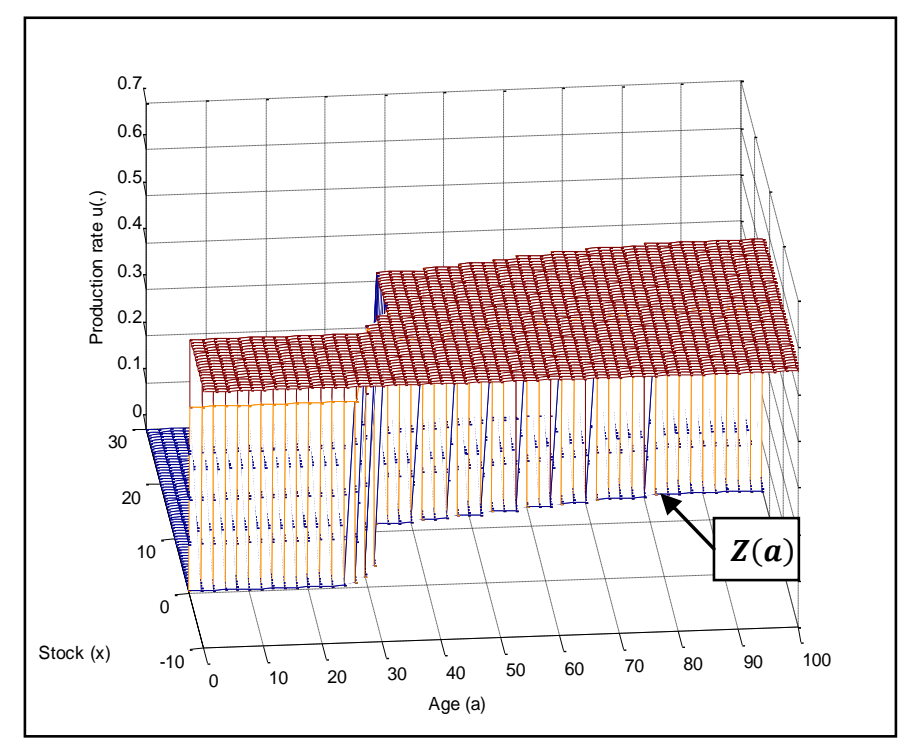

Figure 5. Production policy of the manufacturing system. 


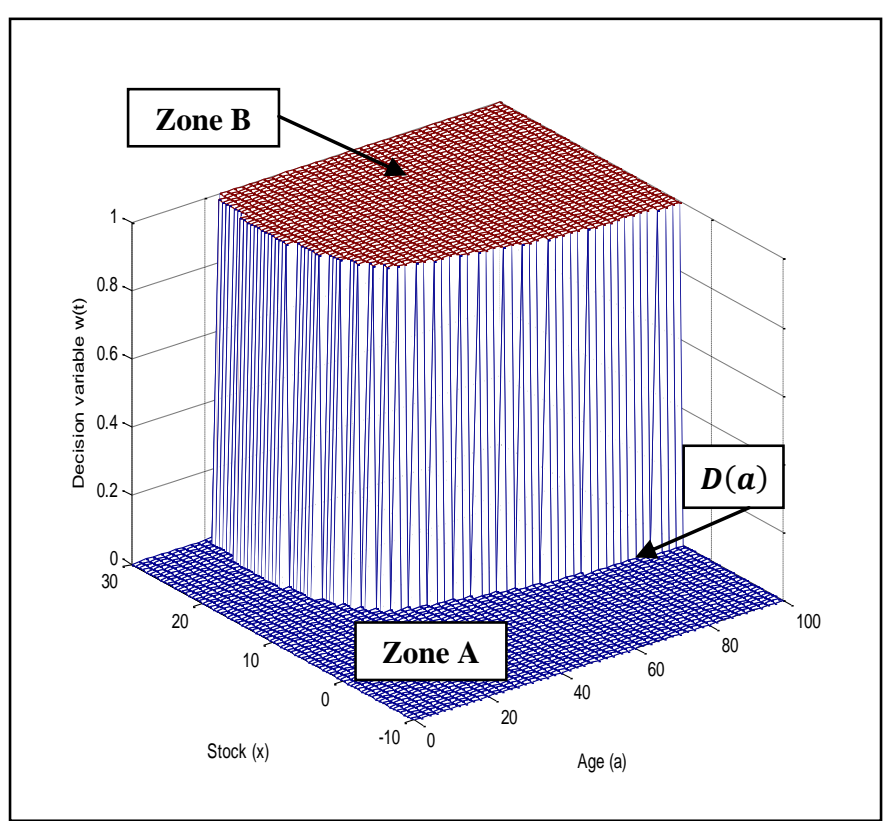

Figure 6. Replacement policy.

switching levels. For every fixed $a$, when $Z(a) \equiv Z$, and $D(a) \equiv D$, the switching curve policy obtained becomes an extension of the so-called hedging point policy, and $Z$ and $D$ are the hedging points. Exploring the impact of aging, we are interested in the functions $Z(a)$ and $D(a)$, which are the optimal threshold levels of production and replacement for each age $a$. In the context of deterioration, we note that the production threshold $Z(a)$ presented in Figure 7 increases progressively. Indeed, when the machine is in its early life period, it is still relatively new and failures are rare. Thus, the inventory level should be maintained almost to a value closer to zero. When the machine is aging and the failure rate increases, building a certain inventory level becomes important, and this level increases with the age of the machine. We note that all values of $w($.$) chosen between \left(10^{-5} \approx 0\right)$ and 1 are permitted in order to extend the domain of $w($.$) to a$ continuous domain, and can apply the theory of convexity, but we know that the optimal solution is reached at the boundary value $10^{-5}$ or 1 , confirming what was mentioned in the section, "Properties of the value function and optimality conditions".

In Figure 7, the intersection between the production threshold $Z(a)$ and the replacement trace $D(a)$ defines the feasible zone $C$, where the manufacturing system resides. This zone recommends optimally, the age of the machine and the necessary stock level when the machine should be replaced. Thus, the replacement policy can be rewritten as follows:

$$
w^{*}(1, x, a)=\left\{\begin{array}{lc}
w_{\max } & \text { if }(x(t), a(t)) \in \text { zone } C \\
w_{\min } & \text { otherwise }
\end{array}\right.
$$

with $C=A \cap B$ where states variables $(x(t), a(t))$ are the parameters of zone C. We note that the point $s$ highlighted on Figure 7 is a point

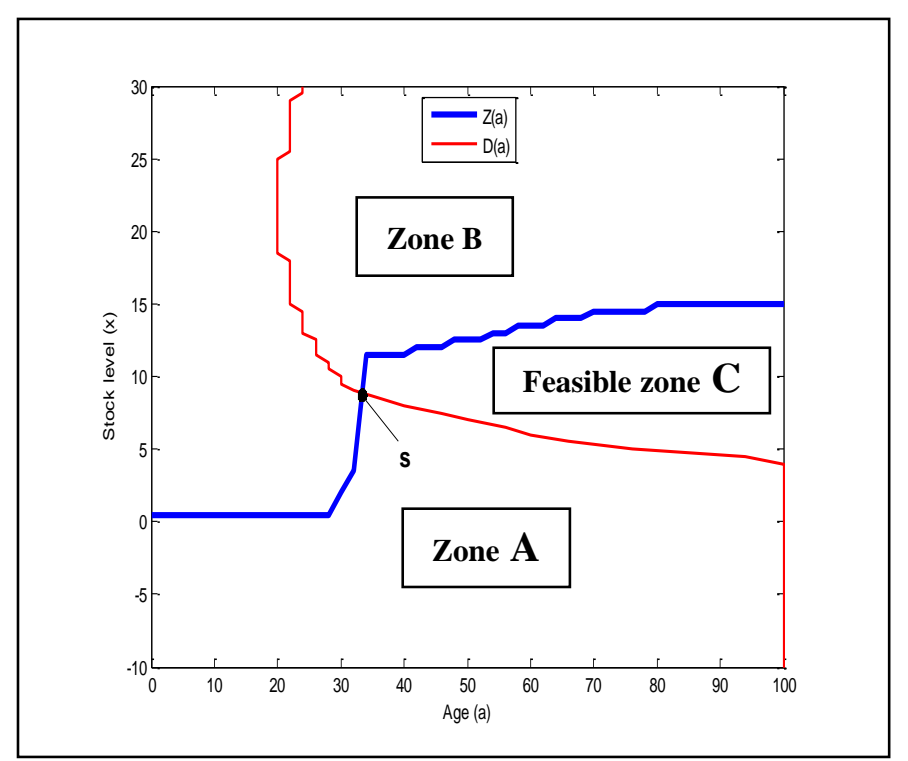

Figure 7. Production threshold and replacement trace.

where the production threshold $Z(a)$ intersects with the replacement trace $D(a)$, and this is a recommended point for machine replacement.

In the next section, we will confirm the obtained structure of the control policies through a sensitivity analysis.

\section{Sensitivity analysis}

The obtained control policies are validated through a sensitivity analysis by varying some parameters of the model. We analyze the behaviour of the production threshold $Z($.$) and$ the replacement trace $D($.$) by varying the following$ parameters: the costs of backlog, surplus, production and replacement, the mean time to repair and the mean time to replace. In the following cases of the variation of parameters, we can note that the symbols $s_{1}, s_{2}$ and $s_{3}$ are highlighted on the following figures, in order to illustrate the intersection points of the trace $D($.$) and the production threshold Z($. when the replacement of the machine is recommended for first time. The three intersection points $s_{1}, s_{2}$ and $s_{3}$ correspond respectively to the small, middle, and high values of the parameter which will be varied in the sensitivity analysis.

\section{Backlog cost variation}

The results presented in Figure 8 for three different backlog cost values $c^{-}=100,150$ and 200 show that when the backlog cost increases, the replacement of the machine is more highly recommended. That is because in this situation, the production threshold $Z($.$) must be increased as c^{-}$increases in order to avoid shortages during periods of future failures of the machine, and to reach this level, the machine must produce 
more parts at its maximum production rate $u_{\max }$. In other words, the machine takes more time to produce at $u_{\max }$. Thus, the machine will deteriorate more rapidly, and for this reason, we replace it earlier: $a\left(s_{3}\right)<a\left(s_{2}\right)<a\left(s_{1}\right)$. Consequently, this increases the feasible zone $\mathrm{C}$, and we can observe that the variation of the backlog cost is directly linked to the size of the replacement zone.

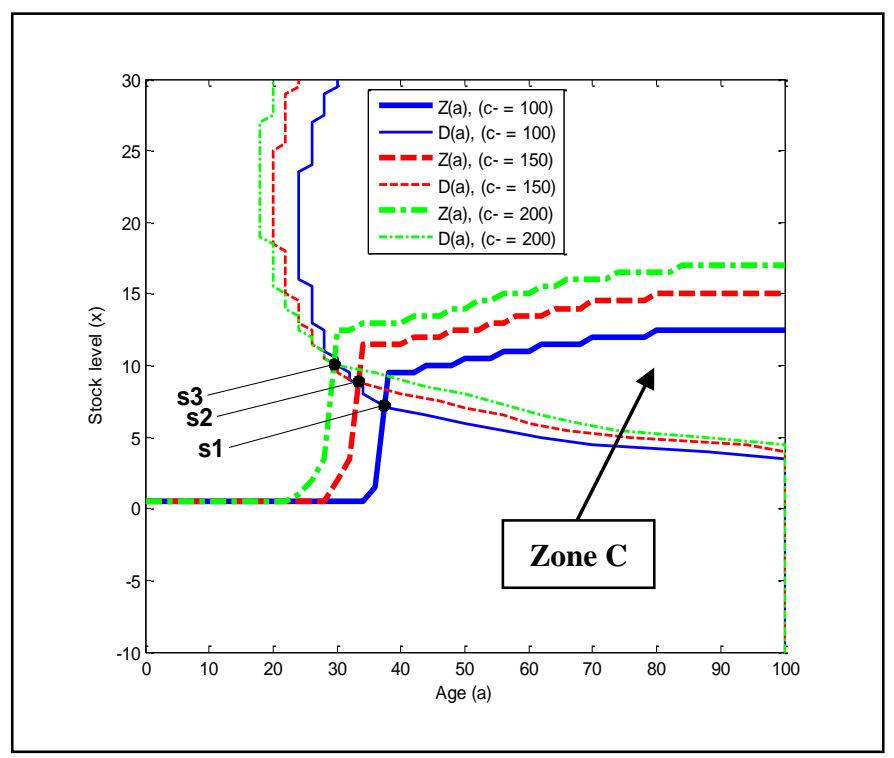

Figure 8. Variation of the backlog cost and its effect on the production and replacement policies.

\section{Inventory cost variation}

As we can see in Figure 9, the next step in the sensitivity analysis is to examine the inventory cost parameter. The values used are $c^{+}=5,10$ and 15 . When the inventory cost $c^{+}$ increases, the replacement of the machine is less recommended. The reason for this is that the production threshold $Z($.$) must be decreased as c^{+}$increases, and in this case, the machine must produce for a shorter period at its maximum production rate $u_{\max }$. Thus, the machine will deteriorate more slowly, and for this reason, we replace it later: $a\left(s_{1}\right)<a\left(s_{2}\right)<a\left(s_{3}\right)$; this means the feasible zone $\mathrm{C}$ is decreased as $c^{+}$increases.

We can conclude from Figures 8 and 9 that the effects of the backlog cost on the production and replacement policies are the inverse of what is seen with the inventory cost. We know that the machine deteriorates with age when it produces the parts. When the threshold increases (in the case of $\mathrm{C}^{-}$ increases or $c^{+}$decreases), the machine produces more parts at its maximum production rate $u_{\max }$ and therefore it is aging rapidly, and it is recommended that it be replaced, and for this reason, the feasible zone $\mathrm{C}$ increases. Conversely, when the threshold decreases (in the case of $c^{-}$decreases or $c^{+}$ increases), the machine produces fewer parts, and ages slowly, and for this reason, the feasible zone $\mathrm{C}$ decreases.

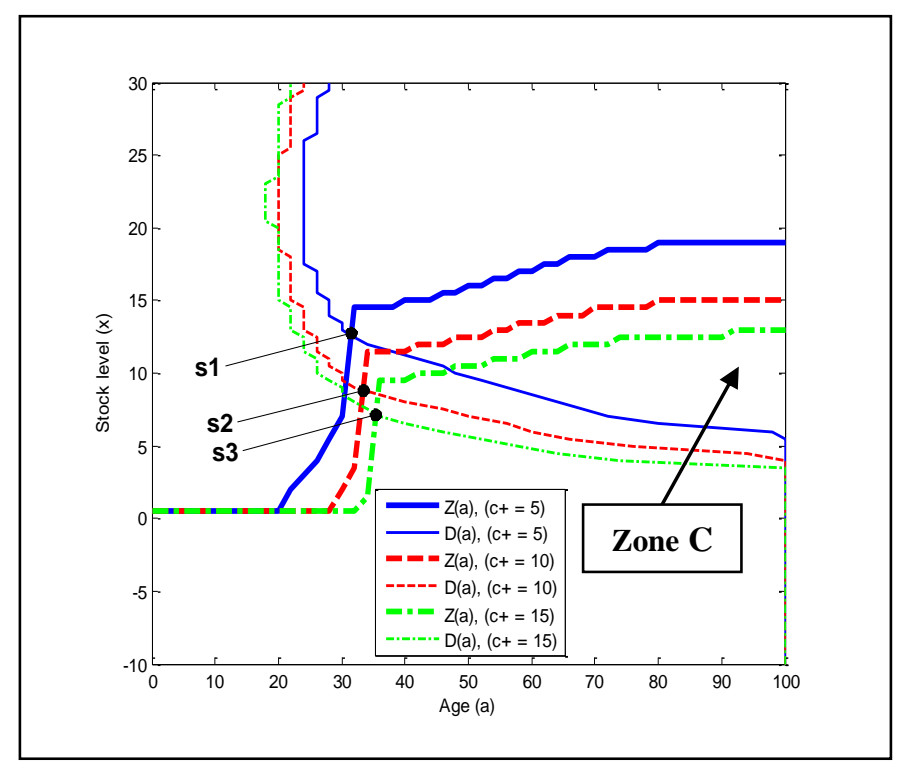

Figure 9. Variation of the inventory cost and its effect on the production and replacement policies.

\section{Production cost rate variation}

We will now analyze the variation of the production cost rate $c_{u}$ for three values $c_{u}=100,500$ and 1000 . The results presented in Figure 10 show that by increasing the production cost rate, the replacement is less recommended, since when the production is more penalized, the production threshold $Z($. must be decreased, and to reach this level, the machine must take less time to produce parts at its maximum production rate $u_{\text {max }}$. Thus, the machine will deteriorate more slowly, and we have to replace it later: $a\left(s_{1}\right)<a\left(s_{2}\right)<a\left(s_{3}\right)$ and the feasible zone $\mathrm{C}$ will decrease.

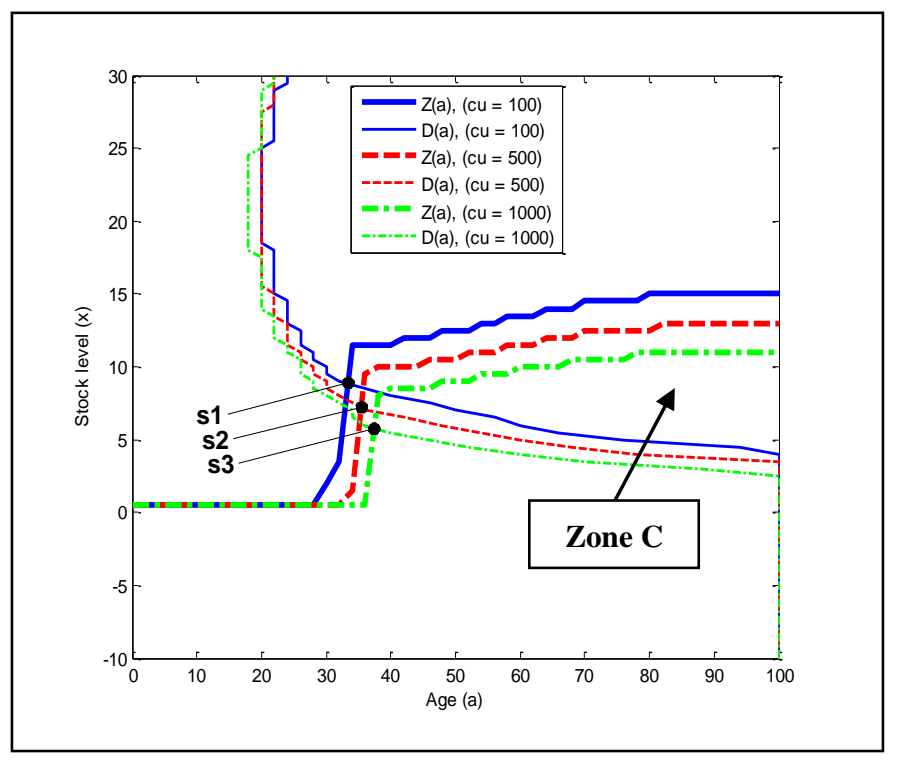

Figure 10. Variation of the production cost rate and its effect on the production and replacement policies. 


\section{Replacement cost variation}

Now, we illustrate the effect of the variation of the replacement cost $c_{0}$ on the production and replacement policies. As we can see from Figure 11, with three different cases, as $c_{0}=1500,3000$ and 4500 , when the replacement cost increases, replacement is less recommended. The reason is that the cost to replace the machine is higher, and it is preferable to keep it longer. That is why we replace the machine later: $a\left(s_{1}\right)<a\left(s_{2}\right)<a\left(s_{3}\right)$. However, with respect to the production policy, the replacement costs have not reported any influence on the production threshold $Z($.$) when$ the machine is at its early stage of life. The change will occur only before entering in the replacement zone, and the system will build a certain stock level to hedge against shortages and to continue to meet customer demand. Once the decision to send the machine for replacement is made, it seems logical that the behaviour of the production threshold $Z($.$) over age$ remains the same (as observed in all three cases from Figure 11 ), because the mean time to replacement is the same in all three cases. Finally, it can be seen that the feasible zone C decreases as the replacement cost increases.

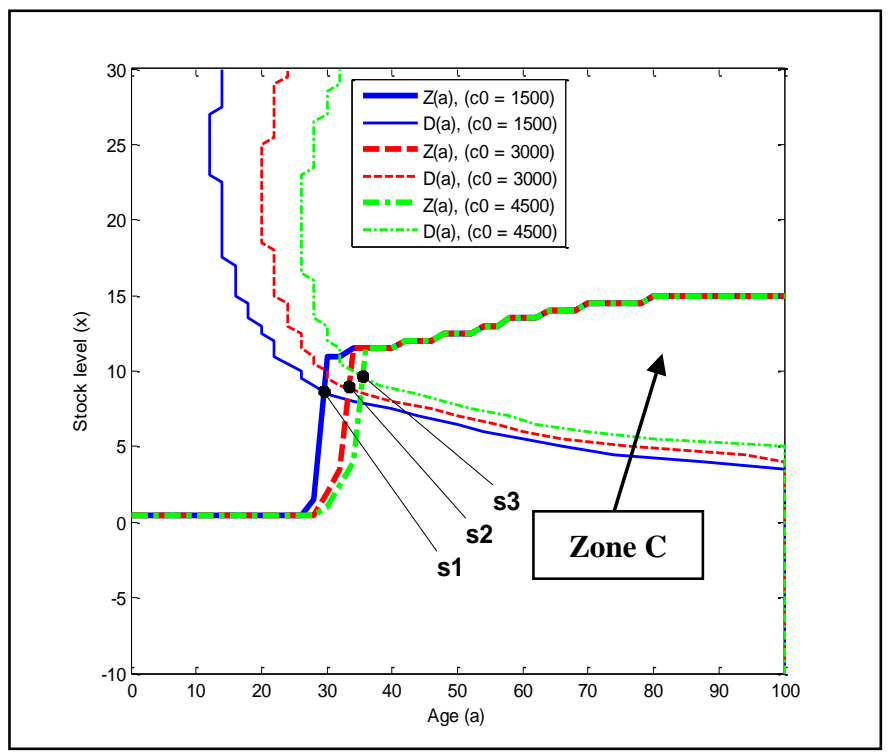

Figure 11. Variation of the replacement cost and its effect on the production and replacement policies.

\section{Mean time to repair variation}

We now discuss the variation of the mean time to repair MTTR for three values, $M T T R=15,20$ and 25. From Figure 12, we can observe that when the MTTR increases, replacement is more highly recommended. The reason is that the machine becomes less available, and to continue to meet customer demand, the production threshold $Z($.$) must be increased;$ however, to be able to do that, the machine must produce more parts at its maximum production rate $u_{\max }$. Thus, we will have a more rapid deterioration of the machine, which is why we replace the machine earlier. We conclude that the age at the intersection point is $a\left(s_{3}\right)<a\left(s_{2}\right)<a\left(s_{1}\right)$, and the feasible zone $\mathrm{C}$ will be increased.

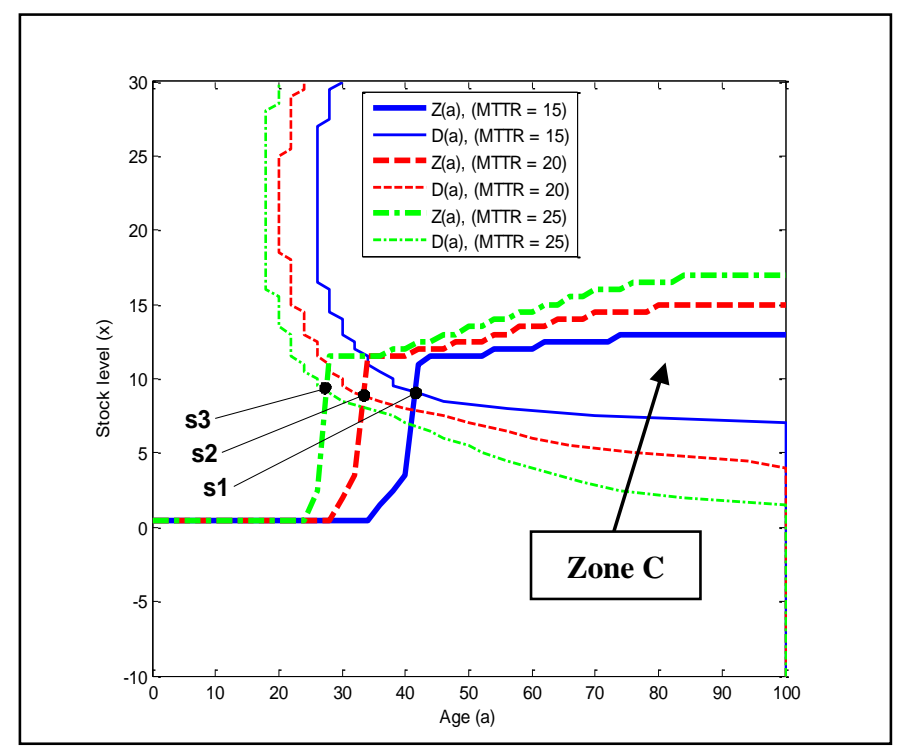

Figure 12. Variation of the mean time to repair and its effect on the production and replacement policies.

\section{Mean time to replacement variation}

To complete the sensitivity analysis, we study the variation of the mean time to replacement MTTRP, for the values 10,14 and 18. We know that the availability of the machine decreases when more time is needed to replace it. As we observe in Figure 13, when the MTTRP increases, replacement is less recommended. The reason is that when it takes time to replace the machine, for the optimal control policy, it is preferable to keep it longer. Thus, we replace the machine later with the age at the intersection point $a\left(s_{1}\right)<a\left(s_{2}\right)<a\left(s_{3}\right)$. Once the decision to send the machine for replacement is made, the machine must first produce more parts at its maximum production rate $u_{\max }$ to hedge against periods of non-production, when the MTTRP increases. Thus, the replacement will be performed with a higher age, and with a higher stock level. In this case, the feasible zone $\mathrm{C}$ will be decreased. We note that the MTTRP has the same effect on the replacement policy as the replacement cost $c_{0}$.

\section{Discussions}

The numerical results presented in this paper show that the control policy for the considered manufacturing system is not a traditional hedging point policy, but rather, is a modified one when the machine deteriorates with age, and the repair activities are as-bad-as-old. This policy is of hedging point type in the sense that it is fully characterized by a curve within a domain of two parameters - the stock level $x$ and the age $a$. We can conclude from the sensitivity analysis presented that the structure of the control policy obtained by our proposed approach is maintained when the parameters of the system 


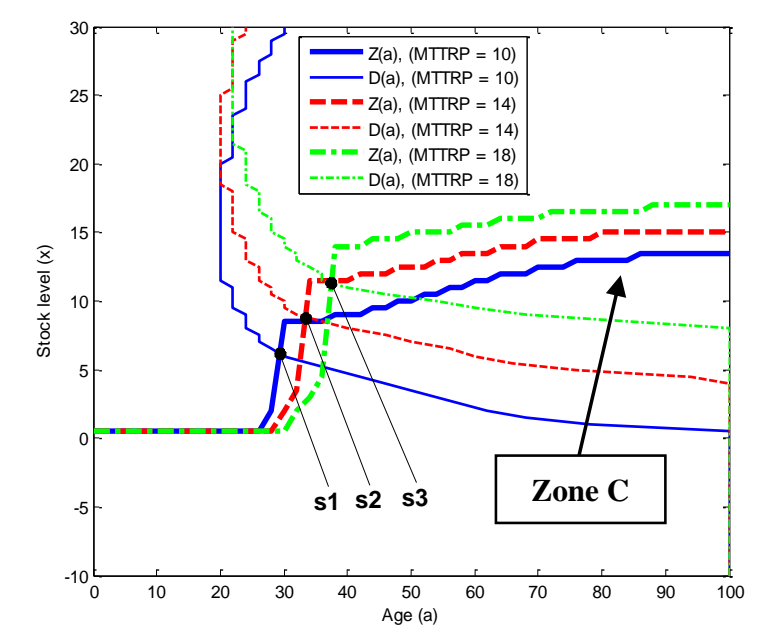

Figure 13. Variation of the mean time to replace the machine and its effect on the production and replacement policies.

vary. Incorporating the new production cost factor in the cost function leads to a reduction of the inventory level while continuing to meet the customer demand, and keeps the machine longer within a low cost level, because it is aging more slowly. The results presented were obtained with the semi-Markovian model because the simpler (Markovian) model is not appropriate for systems subject to deterioration caused by machine aging. A careful analysis of applicability of optimization techniques based on a numerical solution of HJB equations was performed. The production and replacement policies are defined simultaneously by the function $Z(a)$ and $D(a)$, the production threshold trace and the replacement trace, respectively. These two traces, and their intersection point in particular, determine the feasible zone $\mathrm{C}$ and the recommended stock and age levels, at which the decision regarding the machine replacement has to be made.

\section{Conclusion}

In this paper, we determine simultaneously the optimal production and replacement strategies for the case of one machine and one product subject to random failures and repairs. A stochastic optimization model in continuous time has been developed. The considered manufacturing system is under machine's age deterioration with the combined effect of imperfect repairs and AGAN-replacement. By penalizing the production rate, we can explore and observe the impact of the machine aging on the optimal control strategies (production (rate) and replacement policies). From our results, it seems reasonable to incorporate the production parameter into the process of finding optimal control policies in order to get a better solution. This work is strictly related to the age phenomenon, and provides a mathematically accurate analysis of the problem at hand in order to ensure the convergence of numerical methods based on the convexity of two-dimensional $(u, w)$ controls. Since the machine is not new after repair activities, a semi-Markov decision process has to be used in describing its dynamics, which seems more realistic in practice. The optimality conditions were developed in the form of the HJB equations using the stochastic optimal control and dynamic programming approach. The solution of the stochastic control problem is obtained using numerical methods. An extensive sensitivity analysis is performed to validate the structure of the obtained control policies. Finally, an extension of this model to the case of several machines remains to be done, and is a part of our ongoing research.

\section{References}

1. Pham H and Wang H. Imperfect maintenance. Eur J Oper Res 1996; 94: 425-438.

2. Chiu YS, Tseng CY, Liu WC, et al. Economic manufacturing quantity model with imperfect rework and random breakdown under abort/resume policy. Proc IMechE Part B: Journal of Engineering Manufacture 2009; 223: 183-194.

3. Phelps RI. Optimal policy for minimal repair. J Oper Res Soc 1983; 34: 425-427.

4. Kijima M, Morimura H and Suzuki Y. Periodical replacement problem without assuming minimal repair. Eur J Oper Res 1988; 37: 194-203.

5. Makis V and Jardine AKS. A note on optimal replacement policy under general repair. Eur J Oper Res 1993; 69: 75-82.

6. Love CE, Zitron MA and Zhang ZG. An SMDP approach to optimal repair/replacement decisions for systems experiencing imperfect repairs. J Qual Maint Eng 1998; 4: 131-149.

7. Love CE, Zhang ZG, Zitron MA, et al. A discrete semi-Markov decision model to determine the optimal repair/replacement policy under general repairs. Eur J Oper Res 2000; 125: 398409.

8. Zhang YL. A bivariate optimal replacement policy for a repairable system. J Appl Probab 1994; 31: 1123-1127.

9. Rishel R. Control of systems with jump Markov disturbances. IEEE Trans Autom Control 1975; 20: 241-244.

10. Olsder GJ and Suri R. Time optimal control of parts-routing in a manufacturing system with failure prone machines. In: Proceedings of 19th IEEE Conference on Decision and Control. New Mexico: Alburquerque, 1980, pp.722-727.

11. Kimemia $\mathbf{J}$ and Gershwin SB. An Algorithm for the Computer Control of a Flexible Manufacturing System. IIE Trans 1983; 15: 353-362.

12. Boukas EK and Haurie A. Manufacturing flow control and preventive maintenance: a stochastic control ppproach. IEEE Trans Autom Control 1990; 33: 1024-1031.

13. Kushner HJ and Dupuis P. Numerical Methods for Stochastic Control Problems in Continuous Time. New York: SpringerVerlag, 1992.

14. Yan HS. Hierarchical stochastic production planning with delay interaction and demand constraints. Proc IMechE Part B: Journal of Engineering Manufacture 2001; 215: 1603-1617.

15. Yan HS. Practical solution approaches to solve a hierarchical stochastic production planning problem in a flexible automated workshop in China. IIE Trans 2003; 35: 103-115.

16. Nodem FI, Kenné JP and Gharbi A. Production planning and repair/replacement switching policy for deteriorating manufacturing systems. Int J Adv Manuf Technol 2011; 57: $827-840$. 
17. Gomez H, Gharbi A and Kenné JP. Joint control of production, overhaul and preventive maintenance for a production system subject to quality and reliability deteriorations. Int J Adv Manuf Technol 2013; 69: 2111-2130.

18. Kenné JP and Boukas EK. Hierarchical control of production and maintenance rates in manufacturing systems. J Qual Maint Eng 2003; 9: 66-82.

19. Kenné JP and Nkeungoue LJ. Simultaneous control of production, preventive and corrective maintenance rates of a failure-prone manufacturing system. Appl Numer Math 2008; 58: $180-194$.

20. Zio E. Computational Methods for Reliability and Risk Analysis, Series in Quality, Reliability and Engineering Statistics. Singapore: World Scientific, 2009.

21. Ross SM. Introduction to Probability Models. New York: Academic Press, 2003.

22. Kenné JP, Boukas EK and Gharbi A. Control of production and corrective maintenance rates in a multiple-machine, multipleproduct manufacturing system. Math Comput Modell 2003; 38: 351-365.

23. Sethi SP, Zhang $\mathrm{H}$ and Zhang Q. Average-Cost Control of Stochastic Manufacturing Systems, in series: Stochastic Modelling and Applied Probability. New York: Springer, 2005.

24. Yan $\mathrm{H}$ and Zhang Q. A numerical method in optimal production and setup scheduling of stochastic manufacturing systems. IEEE Trans Autom Control 1997; 42: 1452-1455.

25. Akella R and Kumar PR. Optimal control of production rate in a failure prone manufacturing system. IEEE Trans Autom Control 1986; 31: 116-126.

26. Bielecki $\mathrm{T}$ and Kumar PR. Optimality of Zero-Inventory Policies for Unreliable Manufacturing Systems. Oper Res 1988; 36: 532-541.

\section{Appendix 1}

\section{Notation}

$c($.$) \quad production cost function$

$c_{r} \quad$ repair cost rate of the machine

$c_{u} \quad$ production cost rate

$c_{0} \quad$ replacement cost of the machine

$c^{+} \quad$ inventory cost

$c^{-} \quad$ backlog cost

$d \quad$ demand rate of customers

$G($.$) \quad instantaneous cost function$

$J($.$) expected discounted cost function$

$Q($.$) \quad transition rate matrix$

$q_{\alpha \beta}($.$) transition rate from mode \alpha$ to mode $\beta$

$u_{\max }$ maximum production rate of the manufacturing system

$u(t) \quad$ production rate of the manufacturing system at time $t$

$x(t) \quad$ stock level at time $t$

$v($.$) \quad value function$

$\xi(t) \quad$ stochastic process of the system at time $t$

$\rho \quad$ discount rate

\section{Appendix 2}

Optimality conditions and numerical approach
The value function $v(\alpha, x, a)$ defined in equation (18) represents the value of the total cost function described by equation (16), and provides the viscosity solution that satisfies the HJB equations (also called optimality conditions). These conditions are necessary and sufficient for an optimum. If we can solve the HJB equations to obtain the value function $v(\alpha, x, a)$, then we can derive the optimal controls $(u, w)$ that achieve the minimum cost. Bellman's optimality principle requires that the optimal decision in a step be independent of the choice of optimal decisions of the previous steps. We assume that it is possible to determine the optimal trajectory in the time interval $[t, \infty]$. If $v(., t)$ represents the optimal costto-go function at time $t$, then equation (18) takes the form of equation (25).

We apply the principle of dynamic programming on the optimal control problem when the system is in the $\alpha(t), x(t)$ and $a(t)$ states at time $t$. We affirm that if the control is optimal in the time interval $[t, \infty]$, with the initial conditions $\alpha(t), x(t)$ and $a(t)$, then it is also optimal in the time interval $[t+\delta t, \infty]$, with initial conditions $\alpha(t+$ $\delta t), x(t+\delta t)$ and $a(t+\delta t)$ at time $t+\delta t>t$. The value function $v($.$) between t$ and $t+\delta t$ is replaced by its expression from equation (26). The integral in the interval $[t+\delta t, \infty]$ is the value function $v(\alpha(t+\delta t), x(t+\delta t), a(t+$ $\delta t), t+\delta t)$, the discount factor is transformed as $e^{-\rho(s-t)} \mid s=$ $t+\delta t)=e^{-\rho \delta t}$. In this case, the value function $v($.$) can be$ represented by equation (27). Since $G($.$) is a continuous$ function and treated as constant in the interval $t \leq s \leq t+\delta t$, the discount factor over $\delta t$ is $e^{-\rho \delta t}=1-\rho \delta t+o(\delta t)$ and its integral in the time interval $t \leq s \leq t+\delta t$ is given by equation (28).

Assuming that $v($.$) is differentiable, we can apply the$ Taylor series expansion and using the conditional expectation operation $\tilde{E}$ (e.g. for any function $H(\alpha), \tilde{E}\{H(\alpha(t+\delta t))\}=$ $E\{H(\alpha(t+\delta t)) \mid \alpha(t)\})$, we obtain the equation (29).

Now, we expand the conditional expectation $\tilde{E}\{H(\alpha(t+$ $\delta t))\}=H(\alpha(t))+\sum_{j} H(j) \lambda_{j \alpha(t)} \delta t+o(\delta t)$, where the term $o(\delta t)$ denotes the rest in Taylor expansion and is negligible as compared to $\delta t$. After standard transformations, we find that the function $v(., t)$ satisfies the equations of dynamic programming so-called Hamilton-Jacobi-Bellman equations, and represented by equation (30). By considering the stationary regime $v(\alpha, x, a, t) \rightarrow v(\alpha, x, a)$ as $t \rightarrow \infty$, and $\partial v / \partial t \rightarrow 0$, we finally get the HJB equations in the form (31).

We now indicate how to implement Kushner's approach to solve the HJB equations (31) numerically. This numerical method is based on the finite difference approximations and policy improvement technique, and is described in Kushner and Dupuis ${ }^{13}$ as well as also in Yan and Zhang. ${ }^{24}$ It consists in using an approximation for the gradient of the value function based on the numerical scheme of finite differences. Let $h_{x}$ and $h_{a}$ denote the length of the finite difference intervals of the state variables $x$ and $a$. Hence, the value function $v(\alpha, x, a)$ is approximated by $v^{h}(\alpha, x, a)$ and the partial derivatives of the first-order $\partial v(\alpha, x, a) / \partial x$ and $\partial v(\alpha, x, a) / \partial a$ are described in 
equations (32) and (33). The HJB equations (31) can be written in the form of (34), where $q_{\alpha \alpha}=-\sum_{\alpha \neq \beta} q_{\alpha \beta}$ and $\Gamma^{h}(\alpha)$ is the numerical control grid. The discrete dynamic programming equations (34) for the three modes of the system, give the following three equations (35) to (37).

$$
\begin{aligned}
& v(\alpha(t), x(t), a(t), t)=\min _{\substack{u(s), w(s) \\
t \leq s \leq \infty}} E\left\{\int_{t}^{\infty} e^{-\rho(s-t)} G(\alpha(s), x(s), a(s), u(s), w(s), s) d s \mid \alpha(t), x(t), a(t)\right\} \\
& v(\alpha(t), x(t), a(t), t)=\min _{\substack{u(s), w(s) \\
t \leq s \leq \infty}} E\left\{\begin{array}{l}
\int_{t}^{t+\delta t} e^{-\rho(s-t)} G(\alpha(s), x(s), a(s), u(s), w(s), s) d s \mid \alpha(t), x(t), a(t) \\
+\int_{t+\delta t}^{\infty} e^{-\rho(s-t)} G(\alpha(s), x(s), a(s), u(s), w(s), s) d s \mid
\end{array}\right\} \\
& v(\alpha(t), x(t), a(t), t)=\min _{\substack{u(s), w(s) \\
t \leq s \leq t+\delta t}} E\left\{\begin{array}{l}
\int_{t}^{t+\delta t} e^{-\rho(s-t)} G(\alpha(s), x(s), a(s), u(s), w(s), s) d s \mid \alpha(t), x(t), a(t) \\
+e^{-\rho \delta t} v(\alpha(t+\delta t), x(t+\delta t), a(t+\delta t), t+\delta t)
\end{array} \mid\right. \\
& \int_{t}^{t+\delta t} e^{-\rho(s-t)} d s=\frac{-1}{\rho}\left(e^{-\rho(t+\delta t-t)}-e^{-\rho(t-t)}\right)=\frac{-1}{\rho}\left(e^{-\rho \delta t}-1\right)=\frac{-1}{\rho}(1-\rho \delta t-1)+o(\delta t)=\delta t+o(\delta t) \\
& v(\alpha(t), x(t), a(t), t)=\min _{u(t), w(t)} \tilde{E}\left\{\begin{array}{c}
G(\alpha(t), x(t), a(t), u(t), w(t)) \delta t+(1-\rho \delta t) \\
{\left[v(\alpha(t+\delta t), x(t), a(t), t)+v_{t}(.) \delta t+v_{x}(.) \delta x+v_{a}(.) \delta a\right]+o(\delta t)}
\end{array}\right\} \\
& \rho v(\alpha, x, a, t)-\frac{\partial}{\partial t} v(\alpha, x, a, t) \\
& =\min _{u(t), w(t)}\left\{G(\alpha, x, a, u, w)+\dot{x} \frac{\partial}{\partial x} v(\alpha, x, a, t)+\dot{a} \frac{\partial}{\partial a} v(\alpha, x, a, t)+\sum_{\beta} q_{\alpha \beta}(.) v\left(\beta, x, \varphi_{a}(\xi, a)\right)\right\} \\
& \rho v(\alpha, x, a)=\min _{(u, w) \in \Gamma(\alpha)}\left\{G(\alpha, x, a, u, w)+\dot{x} \frac{\partial}{\partial x} v(\alpha, x, a)+\dot{a} \frac{\partial}{\partial a} v(\alpha, x, a)+\sum_{\beta} q_{\alpha \beta}(.) v\left(\beta, x, \varphi_{a}(\xi, a)\right)\right\} \\
& \frac{\partial}{\partial x} v(\alpha, x, a)= \begin{cases}\frac{1}{h_{x}}\left(v^{h}\left(\alpha, x+h_{x}, a\right)-v^{h}(\alpha, x, a)\right) & \text { if }(u-d) \geq 0 \\
\frac{1}{h_{x}}\left(v^{h}(\alpha, x, a)-v^{h}\left(\alpha, x-h_{x}, a\right)\right) & \text { otherwise }\end{cases} \\
& \frac{\partial}{\partial a} v(\alpha, x, a)=\frac{1}{h_{a}}\left(v^{h}\left(\alpha, x, a+h_{a}\right)-v^{h}(\alpha, x, a)\right)
\end{aligned}
$$

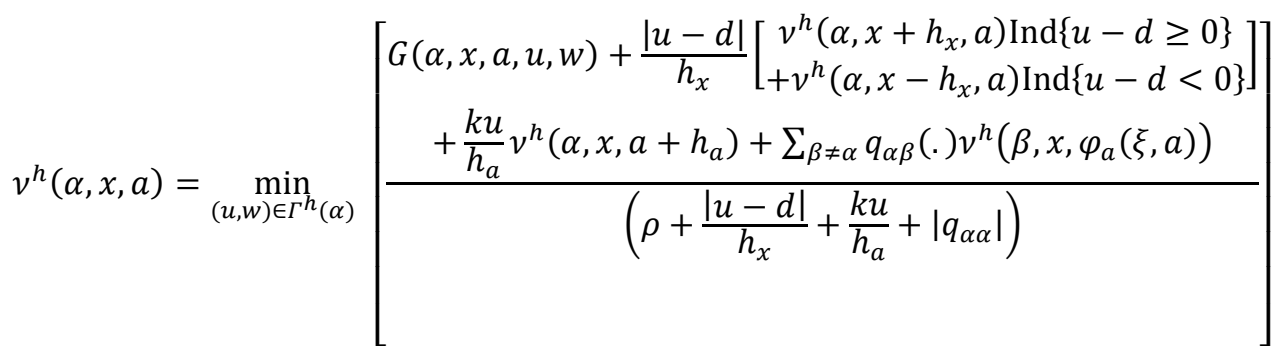


- Mode 1: machine is operational

$$
v^{h}(1, x, a)=\min _{(u, w) \in \Gamma^{h}(1)}\left[\begin{array}{c}
c^{+} x^{+}+c^{-} x^{-}+c_{u} u+\frac{|u-d|}{h_{x}}\left[\begin{array}{c}
v^{h}\left(1, x+h_{x}, a\right) \operatorname{Ind}\{u-d \geq 0\} \\
+v^{h}\left(1, x-h_{x}, a\right) \operatorname{Ind}\{u-d<0\}
\end{array}\right] \\
+\frac{k u}{h_{a}} v^{h}\left(1, x, a+h_{a}\right)+q_{12}(a) v^{h}(2, x, a)+q w v^{h}(3, x, 0)
\end{array}\right]
$$

- Mode 2: machine is under repair

$$
v^{h}(2, x, a)=\min _{(u, w) \in \Gamma^{h}(2)}\left[\frac{c^{+} x^{+}+c^{-} x^{-}+c_{r}+\frac{d}{h_{x}} v^{h}\left(2, x-h_{x}, a\right)+q_{21} v^{h}(1, x, a)}{\left(\rho+\frac{d}{h_{x}}+q_{21}\right)}\right]
$$

- Mode 3: machine is under replacement

$$
v^{h}(3, x, a)=\min _{(u, w) \in \Gamma^{h}(3)}\left[\frac{c^{+} x^{+}+c^{-} x^{-}+c_{0} q_{31}+\frac{d}{h_{x}} v^{h}\left(3, x-h_{x}, a\right)+q_{31} v^{h}(1, x, a)}{\left(\rho+\frac{d}{h_{x}}+q_{31}\right)}\right]
$$

The next lemma shows that the discrete approximation of the value function $v^{h}(\alpha, x, a)$ converges to an exact viscositytype $v(\alpha, x, a)$ solution when the sizes of the grid $h_{x}$ and $h_{a}$ associated with state variables $x$ and $a$ tend to zero.

Lemma 3. Let $v^{h}(\alpha, x, a)$ denotes a solution to HJB equations (35) to (37). Assume that there exist positive constants $C$ and $K>0$ such that:

If $0 \leq v^{h}(\alpha, x, a) \leq C\left(1+|z|^{K}\right)$. Then, $\lim _{h \rightarrow 0} v^{h}(\alpha, x, a)=$ $v(\alpha, x, a)$.
The proof of Lemma 3 is similar to that of Theorem 3 in Yan and Zhang ${ }^{24}$ when replacing $x$ by $z=(x, a)$ and $v(\alpha, x)$ by $v(\alpha, z)$; Hence, we shall not repeat it here.

Finally, some boundary conditions must be imposed when the states are at the border of the finite domain $G_{x a}$ in order to solve the HJB equations (35) to (37) numerically. The effect of this approximation of the boundary conditions on the solution of the original control problem will be negligible (see Boukas and Haurie). ${ }^{12}$ 